\title{
EFFECTS OF DEXAMETHASONE, ADIPOSE DERIVED STEM CELLS AND THEIR EXOSOMES ON INFLAMMATION AND ORAL WOUND HEALING: COMPARATIVE HISTOLOGIC STUDY IN RATS
}

\author{
Ebtehal Mohammed *, Eman Khalil ** and Dina Sabry ${ }^{* * *}$
}

\begin{abstract}
The inflammatory phase of wound healing affects the wound repair as down regulation or up regulation result in delayed healing or scar formation. The dexamethasone is the drug of choice as anti inflammatory, anti edematous and anti emetic in different oral and periodontal surgeries to control post operative pain and edema, however the down regulation of inflammatory phase may affect the wound healing. Adipose derived stem cells (ADSCs), as a part of cell therapy, and exosomes (Exo.), as a part of cell free therapy, revealed promising results in wound healing in preclinical and clinical studies.
\end{abstract}

Materials and Methods: 5o rats were involved in this study. The standardized oral wound was done by scalpel in left cheek then the animals were randomly assigned to 4 groups including gelatin sponge group, dexamethasone group, Exo. group and ADSCs group. The animals were sacrificed at 2,5 and 10 days of healing. The specimens were evaluated histologically (H\&E) stains and inflammatory cells were counted and statistically compared.

Results: regarding inflammatory cell counting, the dexamethasone revealed least inflammatory infiltration followed by Exo.group then ADSCs group and finally, gelatin sponge group. All groups showed signs of healing, However the Exo. group followed by ADSCs showed more condensed collagen formation and increased epithelial thickness than dexamethasone and gelatin sponge groups.

Conclusion: Exo. and ADSCs may accelerate the oral wound healing and overcome the delayed healing that may limit the use of dexamethasone administration as anti inflammatory drug, especially in medically comprised patients as diabetic patients.

KEY WORDS: Oral wound healing, dexamethasone, adipose derived stem cells, exosomes, histologic study, rats

* Lecturer of Oral Medicine, Oral diagnosis and Periodontology, Faculty of Dentistry, Beni Suef University.

** Lecturer of Oral Medicine, Oral diagnosis and Periodontology , Faculty of Dentistry, British University. Egypt.

*** Professor of Biochemistry, Faculty of Medicine, Cairo University. 11562. Cairo. Egypt. 


\section{INTRODUCTION}

Oral wounds could be induced by trauma, iatrogenic factors, oral and periodontal surgeries. The healing of oral wounds, like skin and other wounds in different parts of the body, involves different types of cells and events that should be well-orchestrated. The wound healing proceeds through phases including hemostatic, inflammatory, proliferative and remodeling phase (Enoch $\mathbf{S}$, et al. 2008). The well ordered and balanced inflammatory phase is essential for wound healing and tissue remodeling, not only by the infection prevention, but also by secreting various growth factors enhancing the proliferation of different resident cells as keratinocytes, fibroblasts and endothelial cells in order to secrete extra cellular matrix, collagen deposition and angiogenesis where this role is mainly mediated by macrophages (Larjava H, et al.2012).

Dexamethasone is applied widely in dentistry as premedication to reduce pain and edema after periodontal surgery as open flap debridement (Steffens JP, et al.2010) , mucogonigival surgery (Ligia Nadal Z, et al. 2013) and implant surgery (Bahammam MA, et al.2017) with different protocols of administration (Rajpal J, et al.2015), also they are used in oral and maxillofacial surgeries

(De Oliveira GS, et al.2011) . The anti inflammatory effect of dexamethasone can be attributed to their effect on inflammatory cells by suppressing them in early wound healing events. However, the down regulation of inflammatory phase will adversely affect the wound repair by subsequent suppression of fibroblasts, extra cellular matrix and collagen deposition (Mahmut D, et al.2003).

The absorbable gelatin is considered common hemostatic agent providing many advantages of being inexpensive, absorbable, bio compatible and available. Also they were investigated in many studies a scaffold for different cells in vitro (Anders, J. O. et al.2009), stem cells in vivo (Paganelli, C. et al.2006) and growth factors (Arias-Gallo, J., et al.2013).
The adipose derived stem cells were investigated in wound healing with promising histologic and clinical results in different types of wounds including diabetic wounds, irradiated wounds, burns and ischemic wounds as reviewed by (Mester A, et al.2017). The preclinical studies indicated that they enhanced fibroblasts and keratinocytes proliferation, new blood vessels formation and collagen deposition via secretion of growth factors more than the ability of differentiation and proliferation (Kato Y, et al. 2017). The ADSCs have many advantages over other types of stem cells by being accessible and easily obtained by liposuction , they are abundant in culture, stable karyotyping and safe from transformation (Kolle SF, et al.2013) .

As the suggested effective mechanism of stem cells might be attributed to their "paracrine effect" via secretome or cellular secreted substances, among them extracellular vesicles including apoptotic bodies, and the exosomes "Exo.". They were emerged as "cell free therapy" in regenerative medicine with many advantages over cell therapy being more safe, easily handled and can be dosed as any pharmacological substance (Beer, L.; et al. 2017). Exo. are cellular lipid involved nano vesicles with diameter of $50 \mathrm{~nm}$ and $100 \mathrm{~nm}$ that are secreted to extracellular environment. They contain unique genetic materials and transcription factors that mediate their actions on targeted cells as they are up taken by them (Camussi G, et al. 2010). They revealed promising results in wound healing. However, the exosomes effect differ according to their origin and environment i.e 3D scaffold is better than 2D scaffold. The exact underlying mechanism is not clear, but they had been showed to regulate all wound healing phases as reviewed by (Golchin A, et al.2018). The aim of this study is to investigate the effects of both EXo. and ADSCs on inflammation and wound healing in comparison to dexamethasone which represents the drug of choice in most oral and periodontal surgeries. 


\section{Methodology:}

\section{ADSCs and ADSCs Exosomes preparation:}

The whole procedures were carried out in, Molecular Biology and Tissue Engineering Unit, Department of Medical Biochemistry, Cairo University School of Medicine.

The fat tissues were obtained from inguinal region from 10 weeks old rats $(n=10)$. The excised tissues were hydrolysed for 2 hours by collagenase type II Adipose (Sigma, USA) dissolved in phosphate buffers saline(PBS; Gibco/Invitrogen, Grand Island, New York, USA) at $37^{\circ} \mathrm{C}$. The tissue debris were removed by $2 \mu \mathrm{m}$ strainers followed by centrifugation at $1,000 \mathrm{rpm}$ for $5 \mathrm{~min}$ to form cell pellet that was cultured with a RPMI medium (Gibco BRL, USA), $10 \%$ fetal bovine serum (FBS, Gibco BRL, USA), and humidified in a cell culture incubator containing $5 \% \mathrm{CO} 2$ at $37^{\circ} \mathrm{C}$. The ADSCs were detached with $0.25 \%$ trypsin-EDTA (Gibco BRL, USA) at $80-90 \%$ to be re suspended in other flasks. ADSCs at fourth passage were used. They were characterized in culture by their morphological spindle shaped like cells, by surface marker expression of ADSCs and by flow cytometry (Beckman Coulter). BMSCs were suspended (1x 106 cells $/ \mathrm{ml}$ ) and stained with FITC conjugated monoclonal antibodies, such as CD90 (Biolegend), and CD29 (Biolegend). (Boquest AC,et al. 2006).

Exo. were obtained from supernatants of third passage MSCs (5x106cells $/ \mathrm{ml}$ ) cultured in RPMI deprived of FBS and supplemented with $0.5 \%$ of bovine serum albumin (BSA) (Sigma) (Nassar W, et al.2016).

\section{Study Animals:}

The study was conducted in, Animal house, Kasr El Ainy, Faculty of Medicine, applying the policies and principles established by the Animal Welfare Act and the US National Institutes of Health Guide for Care and Use of Laboratory Animals (http:// oacu. od.nih.gov/ac_cbt/guide3.htm).
The study included 50 Albino Rats weighing 200-250 mg. During the study period, they were housed in wiring cage, 5 rats per cage, under standard conditions, with room temperature was kept $\left(21 \pm 1^{\circ} \mathrm{C}\right)$ and humidity (50-55\%), 12hours dark/light cycle and they were fed the standard rat chow pellets and water available ad libitum .

\section{Wound induction:}

The animals were anesthetized by ketamine $(5 \mathrm{ml})$ and Xylazine $(0.3 \mathrm{ml})$ per animal intra muscularly. The wound was done in the left cheek mucosa according to protocol by (Cavalcante, et al. 2011) using scalpel No $\mathrm{n}^{\mathrm{o}} 15$ scalpel blade to create a standardized wound of $8 \mathrm{~mm}$ length figure $(\mathbf{1}, \mathbf{a})$.

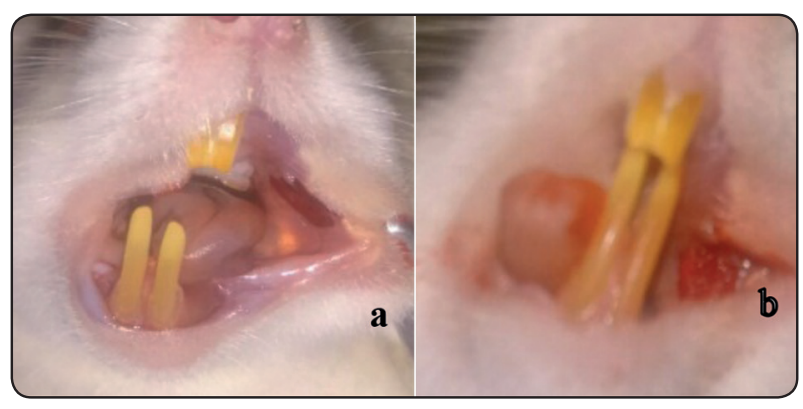

Fig. (1): The figure showing the wound in left cheek (a) and the wound loaded by gel foam (b)

The animals then were randomly allocated into 3 groups (15 rats for each group):

Gel foam group: Received absorbable gelatin sponge (Cutanplast ${ }^{\circledR}$ ) into the wound (Figure 1,b).

Dexamethasone group: Received injectable dexamethasone $8 \mathrm{mg} / 2 \mathrm{ml}$ (Sigmatec-S.A.E) loaded in gelatin sponge into the wound .

Exo. group": Received allogenic ADSCs Exo. loaded on gelatin sponge and applied into the wound .

ADSCs group": Received allogenic ADSCs loaded on gel foam and applied into the wound.

The animals were allowed to heal for 2, 5 and 10 days intervals and they were sacrificed by cervical dislocation. 


\section{Histologic analysis:}

The experimental sites were extracted from the animals after sacrifice at assigned dates, preserved in formalin $10 \%$ for 24 and they were embedded in paraffin. Serial sections (5 micro m) thickness in mucosal surfaces were prepared and stained with hematoxylin and eosin (H\&E) for descriptive analysis and inflammatory cells counting by software (Leica Microsystems, Germany).

\section{Statistical analysis:}

SPSS computer system version 9 was applied to evaluate all the obtained data from the cell counting were statistically evaluated using one -way ANOVA test and The Post hock Tukey. The mean values of data \pm standard deviation were expressed and $\mathrm{P}$ value was calculated to indicate significance.

\section{RESULTS:}

\section{2 days intervals,}

All groups showed diffuse inflammatory reaction with PNL, plasma cell, lymphocytes and macrophages with the gelatin sponge group showed the most diffuse reaction( Figure 2, a) and corticosteroid group showed the least one (Figure 2,b).
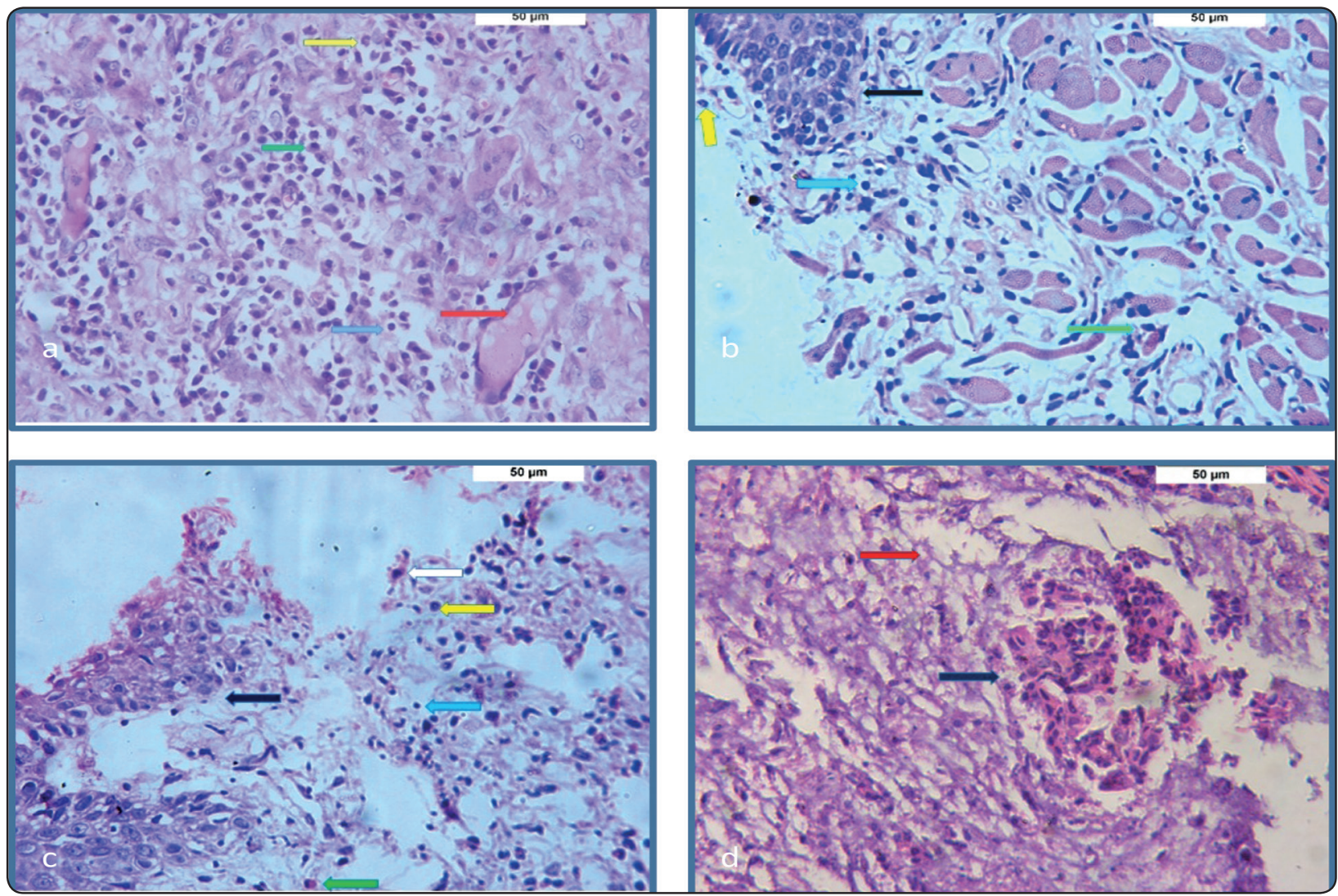

Fig. (2): 2 days intervals, a, Photomicrograph of gelatin sponge group showing numerous inflammatory cells infiltrating the defect area composed of plasma cells (green arrow), lymphocytes (blue arrow) and PNLs (yellow arrow). Dilated blood vessels (red arrow) surrounded by collagen fibrils are also obvious (H\&Ex400), b, Photomicrograph of cortisone group showing regenerated surface epithelium (black arrow) and few inflammatory cells infiltrating the defect area composed of plasma cells (green arrow), lymphocytes (blue arrow) and PNLs (yellow arrow) surrounded by collagen fibrils (H\&Ex400), c, Photomicrograph of Exo. group showing regenerated surface epithelium (black arrow) and numerous inflammatory cells infiltrating the defect area composed of plasma cells (green arrow), lymphocytes (blue arrow), PNLs (yellow arrow) and macrophages (white arrow) surrounded by collagen fibrils. (H\&Ex400). d, Photomicrograph of ADSCs showing regenerated surface epithelium covering a small part of the defect (black arrow). The defect area shows collagen fibers, inflammatory cells and dilated blood vessels (red arrow) (H\&Ex400). 
The regenerated epithelium was evident in corticosteroid group(Figure 2,b), Exo.group (Figure 2,c) and ADSCs group (Figure 2,d). Both Exo. (Figure 2,c) And ADSCs groups showed collagen formation with more condensed fibers in ADSCs group (Figure 2,d).

\section{5 days interval:}

The inflammatory infiltration was less evident than 2 days interval, with more evidence of epithelization and collagen formation.

The inflammatory infiltration in cortisone group (Figure 3,b) is less evident than other groups. However, The thickness of regenerated epithelium and collagen fibers in both Exo.group (Figure 3,c) and ADSCs (Figure3,d) are more than gelatin sponge group (Figure 3,a) and cortisone group (Figure 3,b).
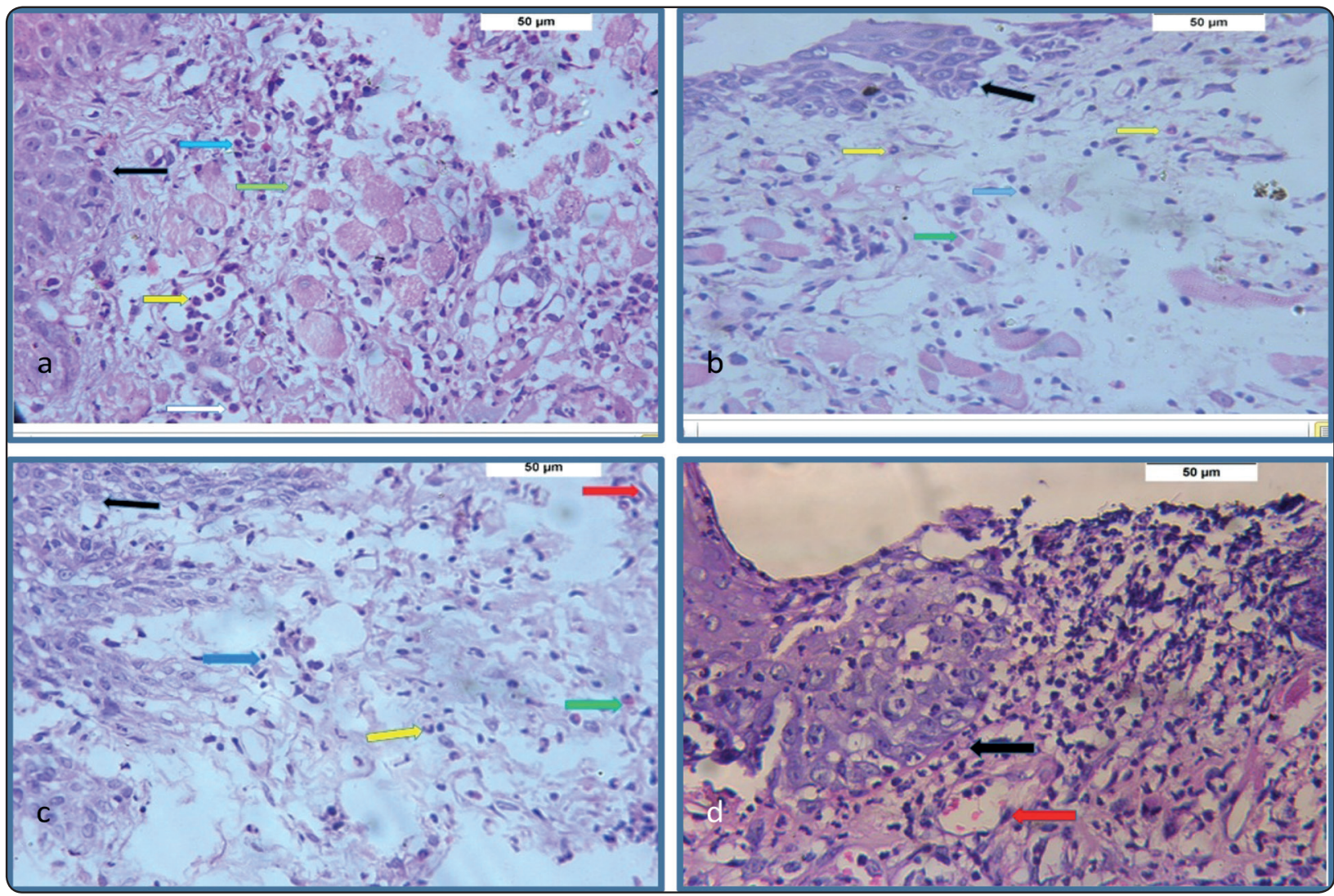

Fig. (3): 5 days interval, a, Photomicrograph of gelatin sponge group showing regenerated surface epithelium (black arrow) Numerous inflammatory cells composed of plasma cells (green arrow), lymphocytes (blue arrow), PNLs (yellow arrow) and macrophages (white arrow) surrounded by collagen fibrils are seen infiltrating the defect area. (H\&E x400). b, Photomicrograph of cortisone group showing regenerated surface epithelium (black arrow) and few inflammatory cells infiltrating the defect area composed of plasma cells (green arrow), lymphocytes (blue arrow) and PNLs (yellow arrows) surrounded by collagen fibrils (H\&Ex400). C, Photomicrograph Exo.group showing regenerated surface epithelium (black arrow) and some inflammatory cells infiltrating the defect area composed of plasma cells (green arrow), lymphocytes (blue arrow) and PNLs (yellow arrow). Dilated blood vessels (red arrow) surrounded by collagen fibers are seen (H\&Ex400).d, Photomicrograph of ADSCs showing regenerated surface epithelium (black arrow) covering a large part of the defect. The defect area shows some inflammatory cells, collagen fibers and dilated blood vessels (red arrow) (H\&Ex400) 


\section{0 days interval,}

The healing was evident in all groups without complete closure of the wound. The cortisone group showed the most uniform healing with the regenerated epithelium is uniform in thickness, collagen fibers are parallel to the surface epithelium and normal blood vessels with least evidence of inflammatory infiltration (Figure 4,b). In Exo. group the regenerated epithelium showed increased thickness and proliferating rete pegs into underlying connective tissue that showed condensed collagen fibers (Figure 4,c) as well as those in ADSCs (Figure 4,d) that also showed dilated blood vessels and together with gelatin sponge group (Figure 4,a) showed inflammatory infiltration.
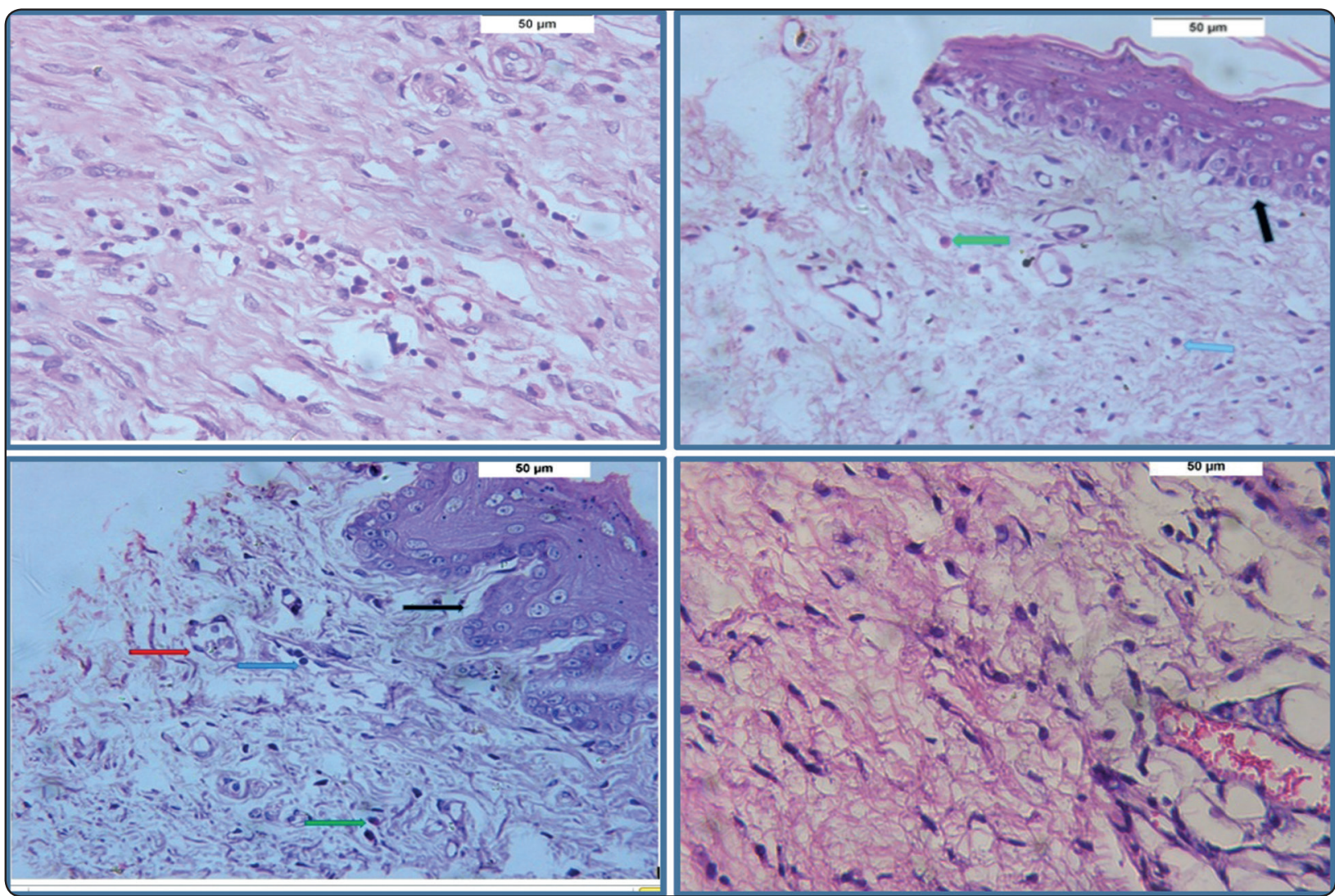

Fig. (4): 10 days interval,a, Photomicrograph of gelatin sponge group showing few inflammatory cells infiltrating the defect area surrounded by bundles of collagen fibers. (H\&Ex400). b, Photomicrograph of cortisone group showing regenerated surface epithelium (black arrow) and very few inflammatory cells infiltrating the defect area composed of plasma cells (green arrow) and lymphocytes (blue arrow) surrounded by collagen fiber bundles (H\&Ex400).c, Photomicrograph of Exo.group showing regenerated surface epithelium (black arrow) and few inflammatory cells infiltrating the defect area composed of plasma cells (green arrow) and lymphocytes (blue arrow) surrounded by dilated blood vessels (red arrow) and collagen fiber bundles (H\&Ex400).d, Photomicrograph of ADSCs group showing few inflammatory cells, dilated blood vessels and many collagen fiber bundles infiltrating the defect area(H\&Ex400) 


\section{Statistical analysis:}

The inflammatory cell count of intragroup was showed in figure (5).

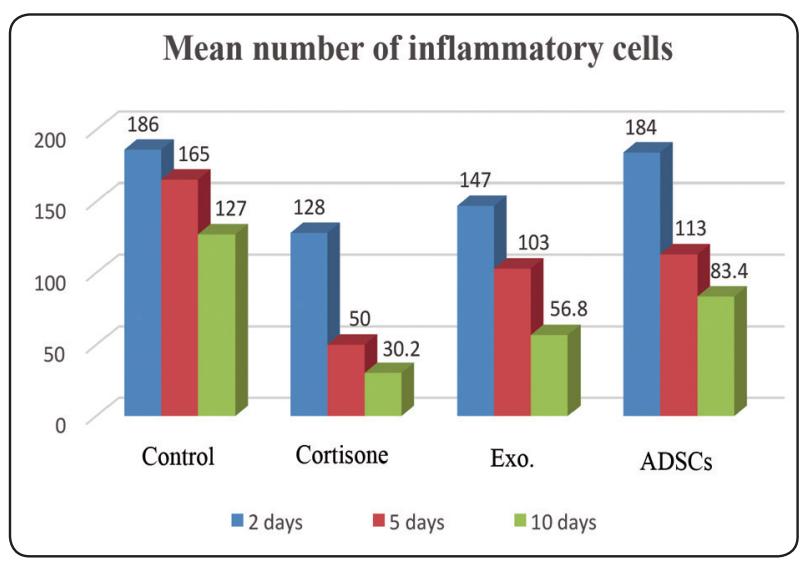

Fig. (5): Bar chart showing mean number of the inflammatory cells of the studied groups after different time intervals
Regarding the inter groups inflammatory cells count, ANOVA test showed significant difference between all groups (table 1).

The Post hock Tukey test (tables 2,3 \&4 ) revealed that the inflammatory cell count in gelatin sponge group was significantly higher than all other groups during 5days and 10days intervals, except ADSCs in 2 days interval. The inflammatory cell count in cortisone group was significantly less than all other groups in all intervals except Exo. group in 2 day interval. The Exo. group was significantly lower than gelatin sponge group and ADSCs in all intervals except in 2 days interval with ADSCs group, While it was significantly higher than cortisone group except in 5 days interval. Finally, the ADSCs group was significantly higher than both cortisone group ,in all intervals, and Exo. group , except in 5 days, While it was significantly lower than gelatin sponge group except in 2 days interval.

TABLE (1): Comparison of mean number of inflammatory cells for the studied groups (ANOVA test):

\begin{tabular}{|c|c|c|c|c|c|}
\hline & control group & Cortisone group & Exo.group & ADSCs group & P Value \\
\hline 2 days & $186 \pm 21.8$ & $128 \pm 13.6$ & $147 \pm 21.9$ & $184 \pm 11.9$ & $\mathrm{P}=0.0002^{*}$ \\
\hline 5 days & $165 \pm 16.8$ & $50 \pm 8.22$ & $103 \pm 9.69$ & $113 \pm 5.36$ & $\mathrm{P}=0.0000^{*}$ \\
\hline 10 days & $127 \pm 11.4$ & $30.2 \pm 7.4$ & $56.8 \pm 12$ & $83.4 \pm 13.9$ & $\mathrm{P}=0.0000^{*}$ \\
\hline
\end{tabular}

Significance is set at $p<0.05$

TABLE (2): Pairwise comparison of mean number of inflammatory cells for the studied groups after 2 days (Tukey Post hoc test):

\begin{tabular}{|c|c|c|c|c|}
\hline Groups & Control group & Cortisone group & Exo. group & ADSCs group \\
\hline Control group & - & $\mathrm{P}=0.0005^{*}$ & $\mathrm{P}=0.0158^{*}$ & $\mathrm{P}=0.9980$ \\
\hline Cortisone group & $\mathrm{P}=0.0005^{*}$ & - & $\mathrm{P}=0.3662$ & $\mathrm{P}=0.0008^{*}$ \\
\hline Exo. group & $\mathrm{P}=0.0158^{*}$ & $\mathrm{P}=0.3662$ & - & $\mathrm{P}=0.0225^{*}$ \\
\hline ADSCs group & $\mathrm{P}=0.9980$ & $\mathrm{P}=0.0008^{*}$ & $\mathrm{P}=0.0225^{*}$ & - \\
\hline
\end{tabular}

Significance is set at $p<0.05$ 
TABLE (3) Pairwise comparison of mean number of inflammatory cells for the studied groups after 5 days (Tukey Post hoc test)

\begin{tabular}{|c|c|c|c|c|}
\hline Groups & Control group & Cortisone group & Exo. group & ADSCs group \\
\hline Control group & - & $\mathrm{P}=0.0000^{*}$ & $\mathrm{P}=0.0000^{*}$ & $\mathrm{P}=0.0000^{*}$ \\
\hline Cortisone group & $\mathrm{P}=0.0000^{*}$ & - & $\mathrm{P}=0.0000^{*}$ & $\mathrm{P}=0.0000^{*}$ \\
\hline Exo. group & $\mathrm{P}=0.0000^{*}$ & $\mathrm{P}=0.0000^{*}$ & - & $\mathrm{P}=0.4856$ \\
\hline ADSCs group & $\mathrm{P}=0.0000^{*}$ & $\mathrm{P}=0.0000^{*}$ & $\mathrm{P}=0.4856$ & - \\
\hline
\end{tabular}

Significance is set at $p<0.05$

TABLE (4) Pairwise comparison of mean number of inflammatory cells for the studied groups after 10 days (Tukey Post hoc test)

\begin{tabular}{|c|c|c|c|c|}
\hline Groups & Control group & Cortisone group & Exo. group & ADSCs group \\
\hline Control group & - & $\mathrm{P}=0.0000^{*}$ & $\mathrm{P}=0.0000^{*}$ & $\mathrm{P}=0.0001^{*}$ \\
\hline Cortisone group & $\mathrm{P}=0.0000^{*}$ & - & $\mathrm{P}=0.0098^{*}$ & $\mathrm{P}=0.0000^{*}$ \\
\hline Exo. group & $\mathrm{P}=0.0000^{*}$ & $\mathrm{P}=0.0098^{*}$ & - & $0.0098^{*}$ \\
\hline ADSCs group & $\mathrm{P}=0.0000^{*}$ & $\mathrm{P}=0.0000^{*}$ & $\mathrm{P}=0.0098^{*}$ & - \\
\hline
\end{tabular}

Significance is set at $p<0.05$

\section{DISCUSSION}

Wound healing includes four phases that are mediated by different cells including the epithelial, resident and recruited inflammatory cells, endothelial and mesenchymal cells (Gurtner, G.C, et al. 2008). The inflammatory phase serve to debride the wound and also to initiate the collagen synthesis, endothelial formation and fibroblasts proliferation, so the regulation of this phase is essential for proper wound healing (Politis C, et al. 2016).

The rodents, including rats and mices, provide a more preferred model for research with similar mucosa and immune subpopulation to human taking into account the ethical and performance difficulties related to non rodent models (Thirion-Delalande C,et al. 2017).

The absorbable gelatin sponge served in many studies as hemostatic agent and scaffold to deliver drugs (Sun W, et al.2013 and cells (Adriana C, et al.2017). In the current study, gelatin sponge group was associated with inflammatory reaction during all intervals more than other groups significantly except ADSCs in 2 days. This inflammatory reaction did not interfere with wound healing. This was similar to results by (Dogru S, et al. 2009) that revealed inflammatory reaction associated with gel foam application in middle ear mucosa. This was explained by mechanism of gel foam absorption that depends on inflammatory tissue reaction to be completed within days to weeks according to size of the sponge (Tomizawa Y.2005)

Dexamethasone is commonly applied drug in management of different types of oral wounds including surgical wounds (Kylmaniemi M, et al.1996) and oral ulcers (Liu C, et al.2012). In the current study the dexamethasone group revealed significantly lower inflammatory infiltration than all other groups in all intervals except Exo. group in 2 days interval, however the Exo.and ADSCs 
showed more collagen formation and increased regenerated epithelium thickness in Exo. group. The similar results of reduction in epithelization and collagen formation by dexamethasone in oral wound healing (Lopes-Rocha R, et al. 2012) and in skin wound (Abreu A M, et al. 2012). This can be explained by the potent anti-inflammatory action in early inflammation with reduction in activation ,proliferation and survival of inflammatory cells, subsequently, the epithelization and fibroplasia are decreased (Nguyen H, et al. 1998). Also, decreased matrix metalloproteinase 8 (MMP 8) secreted by neutrophils with decreased degradation of collagen in wound area which is essential for collagen organization and subsequent collagen deposition (Gutiérrez-Fernández A, et al.2007) .

The exosomes from different cells showed promising results in in wound healing in different animal models, with most extensive studied model is skin wound (Golchin A, et al.2018). In the current study, the Exo.group revealed significantly decreased inflammatory infiltration than both gelatin sponge and ADSCs groups except in 5 days interval with ADSCs, while it was significantly higher than dexamethasone except in 2 days interval. This might be attributed to long action of dexamethasone. As we know, there was no previous study compared the action of dexamethasone and Exo., but in the current study the dexamethasone showed superior anti inflammatory action than Exo., while Exo. showed increased epithelial thickness with many rete pegs indicating good adhesion to underlying connective tissue and more condensed collagen fibers that might be explained by their paracrine effects on targeted cells as they are uptaken by fibroblasts, keratinocytes and endothelial cells enhancing proliferation, extracellular matrix and collagen deposition and angiogenesis (Na YK, et al. 2018).

The ADSCs were investigated in wound healing of different models, specially wounds hard to heal as chronic wounds and they showed promising results due to their paracrine effects on other cells improving the angiogenesis, immune modulation and tissues repair (Bertozzi $\mathbf{N}$, et al. 2017). In the current study, the ADSCs group showed significantly lower inflammatory infiltration than gelatin sponge group except in 2 days. This could be explained by immune modulatory action of ADSCs on stimulation by inflammatory cytokines (Domenis $\mathbf{R}$, ,et al. 2017). While it was significantly higher than both cortisone and Exo. groups in all intervals, except in 5 days with Exo. group. As we know, no previous studies compare the anti inflammatory actions of ADSCs with dexamethasone or their Exo. The ADSCs group showed improved wound healing regarding collagen deposition and epithelization.

\section{CONCLUSION}

The current study compared between dexamethasone as regular therapeutics applied in oral wound healing and ADSCs and their exosomes as recent therapeutics. The dexamethasone revealed the best anti inflammatory action, while the Exo. group and ADSCs showed better wound healing and anti inflammatory action, specially Exo. group in comparison to gelatin sponge group. From these results we can conclude that ADSCs and Exo. may represent a safe alternative for oral wound healing. Also, the gelatin sponge can be used as scaffold to deliver therapeutics locally to wounds. We recommend to combine the exosomes with dexamethasone to over come the retarded healing ability of dexamethasone, specially in comprised healing wounds as diabetic patients.

\section{REFERENCES}

1. Abreu A M, Oliveira D A, Marinho A A,et al. "Effect of Topical Application of Different Substances on Fibroplasia in Cutaneous Surgical Wounds," ISRN Dermatology, 2012 vol. 2012, Article ID 282973, 5 pages, doi. org/10.5402/2012/282973.).

2. Adriana C, , Claudia B, Deise P, et al: Repair of bone defects using adipose-derived stem cells combined with alpha-tricalcium phosphate and gelatin sponge scaffolds in a rat model. Journal of Applied Oral Science, 2017 25(1), 10-19. https://dx.doi.org/10.1590/1678-77572016-0094. 
3. Anders, J. O., Mollenhauer, J., Beberhold, A., Kinne, R. W. \& Venbrocks, R. A. Gelatin-based haemostyptic Spongostan as a possible three-dimensional scaffold for a chondrocyte matrix?: an experimental study with bovine chondrocytes. J Bone Joint Surg Br 2009: 91, 409-416 .

4. Arias-Gallo, J., Chamorro-Pons, M., Avendaño, C. \& Giménez-Gallego, G. Influence of acidic fibroblast growth factor on bone regeneration in experimental cranial defects using spongostan and Bio-Oss as protein carriers. $\mathrm{J}$ Craniofac Surg 2013: 24, 1507-1514 .

5. Bahammam MA, Kayal RA, Alasmari DS, Attia MS, et al: Comparison Between Dexamethasone and Ibuprofen for Postoperative Pain Prevention and Control After Surgical Implant Placement: A Double-Masked, ParallelGroup, Placebo-Controlled Randomized Clinical Trial. J Periodontol. 2017 Jan;88(1):69-77. DOI:10.1902/jop. 2016.160353 .

6. Beer, L.; Mildner, M.; Ankersmit, H.J. Cell secretome based drug substances in regenerative medicine:When regulatory affairs meet basic science. Ann. Transl. Med. 2017 (5) 170.. doi: 10.21037/atm.2017.03.50.

7. Bertozzi N, Simonacci F, and Raposio E: The biological and clinical basis for the use of adipose-derived stem cells in the field of wound healing. Ann Med Surg (Lond). 2017 Jun 23;20:41-48. doi: 10.1016/j.amsu.2017.06.058

8. Boquest AC, Shahdadfar A, Brinchmann JE, Collas P: Isolation of stromal stem cells from human adipose tissue. Methods Mol Biol. 2006;325:35-46. DOI:10.1385/159745-005-7:35).

9. Camussi G, MC Deregibus, S Bruno, V Cantaluppi and L Biancone. Exosomes/microvesicles as a mechanism of cell-to-cell communication. Kidney Int. 2010, 78:838-848.

10. Cavalcante, Galyléia Meneses et al. Experimental model of traumatic ulcer in the cheek mucosa of rats. Acta Cir. Bras. 2011; 26(3). doi.org/10.1590/S0102-86502011000300012.

11. De Oliveira GS, Jr, Almeida MD, Benzon HT, McCarthy RJ. Perioperative single dose systemic dexamethasone for postoperative pain: A meta-analysis of randomized controlled trials. Anesthesiology. 2011;115:575-88.).

12. Dogru, S., Haholu, A., Gungor, A., Kucukodaci, Z., Cincik, H., Ozdemir, T., \& Sen, H. Histologic analysis of the effects of three different support materials within rat middle ear. Otolaryngology-Head and Neck Surgery, 2009 140(2), 177-182. doi:10.1016/j.otohns.2008.10.023.
13. Domenis R, Quaglia S, Cifù A, et al: The immunomodulatory properties of adipose mesenchymal stem cell-derived exosomes are induced by inflammatory cytokines. The FASEB J. 2017 (4),

14. Enoch S, Moseley R, Stephens P\& Thomas D.W.: The oral mucosa: a model of wound healing with reduced scarring. Oral Surgery J (2008) 11-21. doi:10.1111/j.1752248x.2007.00005.

15. Golchin A, Hosseinzadeh S, Ardeshirylajimi A. The exosomes released from different cell types and their effects in wound healing. J Cell Biochem. 2018 Jul;119(7):50435052. doi: $10.1002 /$ jcb.26706.

16. Golchin A, Hosseinzadeh S, Ardeshirylajimi A. The exosomes released from different cell types and their effects in wound healing. J Cell Biochem. 2018 Jul;119(7):50435052. doi: $10.1002 /$ jcb.26706.

17. Gurtner, G.C., Werner, S., Barrandon, Y. \& Longaker, M.T. Wound repair and regeneration. Nature 2008. 453, 314-321.

18. Gutiérrez-Fernández A, Inada M, Balbín M, et al., "Increased inflammation delays wound healing in mice deficient in collagenase-2 (MMP-8)," FASEB Journal,2007 vol. 21, no. 10, pp. 2580-2591.

19. Kato Y, Iwata T, Washio K, et al. Creation and Transplantation of an Adipose derived Stem Cell (ASC) Sheet in a Diabetic Wound-healing Model. J Vis Exp. 2017. doi: 10.3791/54539).

20. Kolle SF, Fischer-Nielsen A, Mathiasen AB, et al. Enrichment of autologous fat grafts with ex-vivo expanded adipose tissue derived stem cells for graft survival: a randomized placebo-controlled trial. Lancet. 2013;382:1113-1120. doi: 10.1016/S0140-6736(13)61410-5

21. Kylmaniemi M, Oikarinen A, Oikarinen K, Salo T. Effects of dexamethasone and cell proliferation on the expression of matrix metalloproteinases in human mucosal normal and malignant cells. J Dent Res. 1996;75(3):919-926).

22. Larjava H, Yang Y, Putnins E, Heino J \& Häkkinen L: Biological agents and cell therapies in periodontal regeneration. Endodontic Topics 2012;26: 18-40.

23. Ligia Nadal Z; Fábio André dos S and Gibson Luiz P. Use of etoricoxib and dexamethasone for postoperative pain prevention and control in mucogingival surgery: a randomized parallel double-blind clinical trial. Braz. J. Oral Sci. [online]. 2013, vol.12, n.4). 
24. Liu C, Zhou Z, Liu G, et al: Efficacy and safety of dexamethasone ointment on recurrent aphthous ulceration. Am J Med. 2012 Mar;125(3):292-301. doi:10.1016/j.amjmed. 2011.09.011.

25. Lopes-Rocha R., Miranda J.L, Lima N.L., Ferreira F.O, et al. Effect of topical propolis and dexamethasone on the healing of oral surgical wounds. Wound Healing Southern Africa. 2012 1(5).

26. Mahmut D; Erol K; Erdogan O; et al: The Effects of Single-Dose Dexamethasone on Wound Healing in Rats. Anesthesia \& Analgesia: 2003 (97): 5 -1377-1380. doi: 10.1213/01.ANE.0000080611.29106.9E.

27. Mester, A., Opincariu, D., Benedek, I., \& Benedek, I.. Stem Cell Therapy in Wound Healing, Journal of Interdisciplinary Medicine, 2017 2(s4), 20-24. doi: https://doi. org/10.1515/jim-2017-0094.

28. Na YK, Ban JJ, Lee M, Im W, Kim M. Wound healing potential of adipose tissue stem cell extract. Biochem Biophys Res Commun. 2017 Mar 25;485(1):30-34. doi: 10.1016/j.bbrc.

29. Nassar W, El-Ansary M, Sabry D, Mostafa MA, et al:: Umbilical cord mesenchymal stem cells derived extracellular vesicles can safely ameliorate the progression of chronic kidney diseases. Biomater Res. 2016 Aug 5;20:21. doi: 10.1186/s40824-016-0068-0.

30. Nguyen H, Lim J, Dresner M. L., and Nixon B., "Effect of local corticosteroids on early inflammatory function in surgical wound of rats," Journal of Foot and Ankle Surgery 1998, vol. 37, no. 4, pp. 313-318.
31. Paganelli, C. et al. Indications on suitable scaffold as carrier of stem cells in the alveoloplasty of cleft palate. $\mathrm{J}$ Oral Rehabil 2006: 33, 625-629 .

32. Politis C, Schoenaers J, Jacobs R and Agbaje JO :Wound Healing Problems in the Mouth. Front. Physiol. 2016. 7:507. doi: 10.3389/fphys.2016.00507.

33. Rajpal J, Arora A, Prasad R, Gupta MM. Preventing postoperative swelling after periodontal surgery. J Oral Res Rev 2015;7:31-4. 10.4103/2249-4987.160193.

34. Steffens JP, Santos FA, Sartori R, Pilatti GL. Preemptive dexamethasone and etoricoxib for pain and discomfort prevention after periodontal surgery: a double-masked, crossover, controlled clinical trial. J Periodontol. 2010 Aug;81(8):1153-60. doi: 10.1902/jop.

35. Sun W, Chen Y, Yuan W. Hemostatic absorbable gelatin sponge loaded with 5-fluorouracil for treatment of tumors. Int J Nanomedicine. 2013;8:1499-506. doi: 10.2147/IJN. S41462.

36. Thirion-Delalande C, Gervais F, Fisch C, Cuiné J, BaronBodo V, Moingeon P, et al. Comparative analysis of the oral mucosae from rodents and non-rodents: Application to the nonclinical evaluation of sublingual immunotherapy products. PLoS ONE 2017 12(9): e0183398. https://doi. org/10.1371/journal.pone.0183398).

37. Tomizawa Y. Clinical benefits and risk analysis of topical hemostats:review. J Artif Organs. 2005;8:137-42. DOI:10.1007/s10047-005-0296-x. 